\title{
Aspectos epidemiológicos e clínicos dos acidentes ofídicos ocorridos nos municípios do Estado do Amazonas
}

\author{
Epidemiological and clinical aspects of snake accidentes in the \\ municipalities of the State of Amazonas, Brazil
}

Célio Campos Borges ${ }^{1}$, Megumi Sadahiro² e Maria Cristina dos Santos ${ }^{3^{*}}$

\begin{abstract}
Resumo No Amazonas, o acidente ofídico é um problema de saúde pública pouco conhecido. Por este motivo, foi realizado um estudo descritivo dos acidentes ofídicos atendidos nas Unidades de Saúde de 34 municípios, um distrito e dois pelotões de fronteira do Estado do Amazonas. As características mais comuns encontradas dentre os pacientes foram: agricultor (50,4\%), do sexo masculino (81,3\%), em idade produtiva $(72,1 \%)$, picado no membro inferior $(88,5 \%)$, por jararaca $(48,6 \%)$ ou surucucu $(46,8 \%)$, na zona rural de seu município $(70,2 \%)$ e que só recebeu atendimento médico em tempo superior a seis horas, após acidente $(57,3 \%)$. As manifestações locais mais freqüentes foram: edema (76,9\%), dor (68,7\%), eritema $(10,2 \%)$ e hemorragia $(9,3 \%)$. Hemorragia $(18,8 \%)$ foi a manifestação sistêmica mais freqüente. $O$ antiveneno foi administrado em apenas $65,9 \%$ dos pacientes. A via mais utilizada foi a endovenosa (52,3\%), sendo relevante 0 uso de vias não mais recomendadas $(47,7 \%)$. O antiveneno administrado, na maioria dos pacientes, foi o antibotrópico (66,7\%). As complicações mais freqüentes foram abcesso $(13,7 \%)$, necrose (12,3\%), infecção secundária (8,3\%), insuficiência renal (2,5\%) e gangrena (2,5\%). Os procedimentos médicos mais usados para o tratamento das complicaçôes foram: drenagem (52,6\%), debridamento (28,9\%), amputação (10,5\%), limpeza cirúrgica $(5,3 \%)$ e diálise peritoneal (2,6\%). A letalidade foi de $1 \%$.
\end{abstract}

Palavras-chaves: Acidente ofídico. Epidemiologia. Clínica. Amazonas.

\begin{abstract}
In the State of Amazonas, accidents with snakes are a public health problem. For this reasons, the objective of this work was to carry out a descriptive study of the snake accidents attended in the health units of 34 municipalities, one district and two border platoons in the State of Amazonas. The characteristics most commonly observed among those involved in snake accidents were: farmers (50.4\%), male (81.3\%), belonging to the working age-group (72.1\%), bitten on an upper limb (88.5\%) by a "jararaca" (48.6\%) or a "surucucu" (46.8\%) in the rural part of the municipality $(70.2 \%)$. The local signs and symptoms most frequently observed in those who received medical care more than 6 hours after the accident (57.3\%) were edema (76.9\%), pain (68.7\%), erithema (10.2\%) and hemorrhage (9.3\%). The systemic manifestation most frequently observed was hemorrhage (18.8\%). Serotherapy was administered in only $65.9 \%$ of patients, the intravenous route being the route most commonly used to administer the antivenin (52.3\%), while other non- recommended routes were widely used. In the majority of patients the antivenin given was antibotropic. The most frequent complications were: abscess $13.7 \%$, necrosis $12.3 \%$, secondary infection $8.3 \%$, renal insufficiency $2.5 \%$ and gangrene $2.5 \%$. The medical procedures most used in the treatment of these complications were drainage $52.6 \%$, debridement $28.9 \%$, amputation $10.5 \%$, surgical cleaning $5.3 \%$ and peritoneal dialysis $2.6 \%$. The fatality rate was $1 \%$.
\end{abstract}

Key-words: Snake bites. State of Amazonas. Epidemiology.

\footnotetext{
1. Bolsista do Programa Institucional de Bolsas de Iniciação Científica PIBIC/FUA/CNPq; 2. Instituto de Dermatologia Tropical e Venereologia Alfredo da Matta (IDTVAM); 3. Fundação Universidade do Amazonas.

Apoio financeiro: Programa Institucional de Bolsas de Iniciação Científica CNPq/FUA; Pró-Reitoria para Extensão da Fundação Universidade do Amazonas

Endereço para correspondência: Prof ${ }^{a}$ Maria Cristina dos Santos. Laboratório de Imunologia, Departamento de Parasitologia, ICB/FUA. Av. Gal. Rodrigo Otávio Jordão Ramos 3000, Bloco A, Mini-Campus, 69077-000 Manaus, AM.

Tel: 5592 644-1295; Fax: 5592 644-1853.

E-mail: dosantos@fua.br

Recebido para publicação em 1/6/98.
} 
Segundo Campbell e Lamar ${ }^{7}$, ocorrem no Estado do Amazonas sete serpentes da família Viperidae (Bothriopsis bilineata, Bothriopsis taeniata, Bothrops atrox, Bothrops brazili, Crotalus durissus, Lachesis muta muta e Porthidium hyoprora), pelo menos, onze espécies da família Elapidae (Micrurus averyi, M. collaris, M. filiformis, M. hemprichii, M. karlschimidti, M. langsdorff, $M$. lemniscatus, $M$. narduccii, $M$. putumayensis, M. spixii e M. surinamensis) e três gêneros da família Colubridae com registro de envenenamento, em humanos (Philodryas viridissimus, Clelia clelia e Erythrolamprus aesculapii) ${ }^{76}$.

Em estudo epidemiológico retrospectivo realizado com 734 pacientes atendidos no Instituto de Medicina Tropical do Amazonas, no período de 1986 a 1992, os quais eram, em sua maioria, procedentes dos municípios circunvizinhos à cidade de Manaus (Iranduba, Careiro da Várzea, Castanho e Rio Preto da Eva), foi observado que as principais espécies de serpentes causadoras de acidentes nesta região foram Bothrops atrox $(76 \%)$ e Lachesis muta muta $(10 \%)^{25}$. Os envenenamentos por estas espécies apresentam sintomatologias semelhantes (edema, dor, hemorragia local e sistêmica, necrose tecidual e insuficiência renal aguda). Existem algumas evidências de que a sintomatologia vagomimética, apresentada nos empeçonhamentos por Lachesis muta muta, possa ser usada como critério diferencial no diagnóstico clínico dos acidentes 121214 . Segundo estudo retrospectivo, apenas $14,3 \%$ dos pacientes acidentados por Lachesis muta muta apresentam sintomas vagomiméticos ${ }^{26}$. Entretanto, iniciativas recentes têm buscado um imunodiagnóstico diferencial para os envenenamentos botrópicos e laquéticos ${ }^{1813}$, visto que são raros os pacientes que trazem o animal causador ${ }^{92025}$, prejudicando a administração de uma soroterapia específica.

Em algumas regiões do Brasil, o Metronidazol e a Heparina estão sendo utilizados, com base em trabalhos inconclusivos, como substitutos dos antivenenos no tratamento dos pacientes acidentados por ofídios ${ }^{172127}$. Boechat e colaboradores ${ }^{4}$ demonstraram que a Heparina, além de não neutralizar a atividade coagulante do veneno de Bothrops atrox, potencializou a sua ação hemorrágica. Em relação ao Metronidazol a eficácia deste medicamento é obtida quando utilizado em dosagens tóxicas ${ }^{21}$. $O$ extrato vegetal denominado "Específico P. Pessoa", que tem seu uso difundido na Amazônia como antiveneno polivalente, não foi capaz de neutralizar as principais atividades do veneno de Bothrops atrox ${ }^{510}$.

Quanto à sazonalidade do Acidente Ofídico, tem sido sustentada a hipótese da influência da precipitação pluviométrica, a qual elevaria os níveis das águas dos rios, fazendo com que as serpentes que habitam as regiões próximas dessas margens se desloquem a procura de terra firme, e com a diminuição do espaço territorial, aumente o contato com o Homem, facilitando a ocorrência dos acidentes ${ }^{1925}$.

O Acidente Ofídico é um problema de saúde pública no Amazonas, sendo importante a realização de estudos epidemiológicos e clínicos para o seu melhor conhecimento. Por este motivo, o presente trabalho descreve os aspectos epidemiológicos e clínicos do Acidente Ofídico no Interior do Estado do Amazonas.

\section{MATERIAL E MÉTODOS}

Fonte de dados. Neste trabalho foram analisados dados epidemiológicos e clínicos levantados dos prontuários de internação dos pacientes vítimas de acidentes ofídicos ocorridos, no interior do Estado do Amazonas, no período de janeiro de 1989 a dezembro de 1996. Para isso, foram avaliadas Unidades de Saúde, que prestam atendimento aos acidentados por animais peçonhentos, dos municípios das seguintes regiões: do Alto Solimões: Benjamim Constant, Tabatinga e Pelotões de Fronteira de: Atalaia do Norte e Santo Antônio do Içá; do Triângulo (Jutaí/Juruá/Solimões): Tefé; do Purus: Lábrea e Pauini; do Juruá: Carauari, Eirunepé e Envira; do Madeira: Apuí, Borba,
Humaitá, Manicoré; do Alto Rio Negro: Barcelos, Santa Isabel do Rio Negro e São Gabriel da Cachoeira; do Rio Negro/Solimões: Autazes, Castanho, Careiro da Várzea, Coari, Iranduba, Manacapuru e Novo Airão; do Médio Amazonas: Itacoatiara, Itapiranga, Maués, Presidente Figueiredo, Rio Preto da Eva, Silves e o distrito de Balbina; Baixo Amazonas Barreirinha, Boa Vista do Ramos, Nhamundá, Parintins, São Sebastião do Uatumã e Urucará (Tabela 1).

Dados Epidemiológicos. Idade, sexo, profissão, naturalidade, procedência segundo zona (rural ou urbana), horário do acidente, segmento corporal picado, serpente causadora 
Tabela 1 - Distribuição dos acidentes ofídicos por Município do Estado do Amazonas.

\begin{tabular}{|c|c|c|}
\hline Municípios & Período & Número de casos \\
\hline Apuí & jan/92 a jul/95 & 28 \\
\hline Autazes & $\mathrm{abr} / 93$ a jul/94 & 34 \\
\hline Barcelos & jan/90 a jan/96 & 39 \\
\hline Barreirinha & jan/92 a dez/94 & 22 \\
\hline Benjamim Constant & jan/90 a dez/96 & 162 \\
\hline Boa Vista do Ramos & jun/91 a jul/95 & 5 \\
\hline Borba & jan/92 a mai/96 & 86 \\
\hline Carauari & jan/92 a jun/96 & 67 \\
\hline Careiro da Várzea & $\ldots$ & $\ldots$ \\
\hline Castanho & abr/92 a jul/94 & 28 \\
\hline Coari & jan/92 a dez/93 & 109 \\
\hline Eirunepé & $\mathrm{jan} / 91$ a fev/96 & 46 \\
\hline Envira & - & - \\
\hline Humaitá & out/93 a jun/96 & 58 \\
\hline Iranduba & $\ldots$ & $\ldots$ \\
\hline Itacoatiara & jan/93 a jan/95 & 98 \\
\hline Itapiranga & out a dez/94 & 3 \\
\hline Lábrea & jan/93 a mai/96 & 33 \\
\hline Manacapuru & jan/90 a jul/94 & 132 \\
\hline Manicoré & mai/93 a set/96 & 75 \\
\hline Maués & jan/91 a jan/95 & 80 \\
\hline Nhamundá & jan/91 a dez/94 & 28 \\
\hline Novo Airão & ago/93 a mai/96 & 19 \\
\hline Parintins & jan/89 a dez/94 & 136 \\
\hline Pauini & out/93 a jun/94 & 4 \\
\hline Pres. Figueiredo & jan/93 a dez/94 & 21 \\
\hline Rio Preto da Eva & $\ldots$ & $\ldots$ \\
\hline Silves & mar a jul/94 & 3 \\
\hline Sta Izabel do R. Negro & out/92 a out/95 & 14 \\
\hline S. Gabriel Cachoeira & $\mathrm{dez} / 93$ a out/95 & 26 \\
\hline S. Sebastião do Uatumã & $\ldots$ & $\ldots$ \\
\hline Tabatinga & set/92 a jul/95 & 96 \\
\hline Tefé & fev/94 a mai/95 & 78 \\
\hline Urucará & jan/91 a dez/94 & 46 \\
\hline Total & & 1576 \\
\hline
\end{tabular}

Fonte: Prontuários de Internação das Unidades de Saúde dos respectivos Municípios do Amazonas

(...) sem informações disponíveis, pois os pacientes são transferidos para outros Municípios

(-) Não foram encontrados os prontuários.

do acidente e a sazonalidade dos acidentes. Em relação aos tipos de serpentes causadoras de acidentes, os dados obtidos basearam-se nas informações dos acidentados ou dos seus acompanhantes, ou na observação, pelos profissionais de saúde, dos sinais e sintomas apresentados pelos pacientes. Portanto, em todos os prontuários de acidente ofídico levantados neste trabalho o reconhecimento da serpente causadora do acidente não foi feito por profissionais especializados.
Dados Operacionais. Tempo decorrido entre 0 acidente e o atendimento médico.

Dados Clínicos. Dor, edema, eritema, equimose, flictena, hemorragia local, hemorragia sistêmica, náusea, vômito, tontura, desmaio, cefaléia, alterações visuais, parestesia local, oligúria, anúria, hipertermia, pressão arterial e outros sintomas (dispnéia, cianose, mialgia, desorientação e dores abdominais).

Dados de tratamento. Soroterapia (teste de sensibilidade, nome do soro administrado, número de ampolas, via de administração do 
soro), as reações de hipersensibilidade aos antivenenos (soros heterólogos) e os outros medicamentos utilizados pelos profissionais de saúde no atendimento dos pacientes acidentados (antibióticos, anti-inflamatórios, analgésicos, antihistamínicos, ansiolíticos, metronidazol, antieméticos, toxóide tetânico, Específico P. Pessoa, vitaminas, hemostáticos, diuréticos, corticóides, adrenalina e outros medicamentos).
O tempo de coagulação sangüínea (TC), tempo de internação, condições de alta dos pacientes, complicações e condutas realizadas para as complicações, também foram analisados.

O programa, para computador, Epiinfo versão 6.0 foi utilizado para o armazenamento e a análise dos dados.

\section{RESULTADOS}

Dados epidemiológicos e clínicos. Do levantamento nas Unidades de Saúde foram obtidos 1.576 prontuários de acidentes ofídicos. Contudo, foram poucos os prontuários que continham todas as informações.
As características mais comuns encontradas dentre os pacientes acidentados por serpentes ( $n=1576)$ foram: ser natural do Amazonas $(89 \%)$, do sexo masculino $(81,3 \%)$, agricultor $(50,4 \%$, Figura 1), em idade produtiva entre 15 a 59 anos

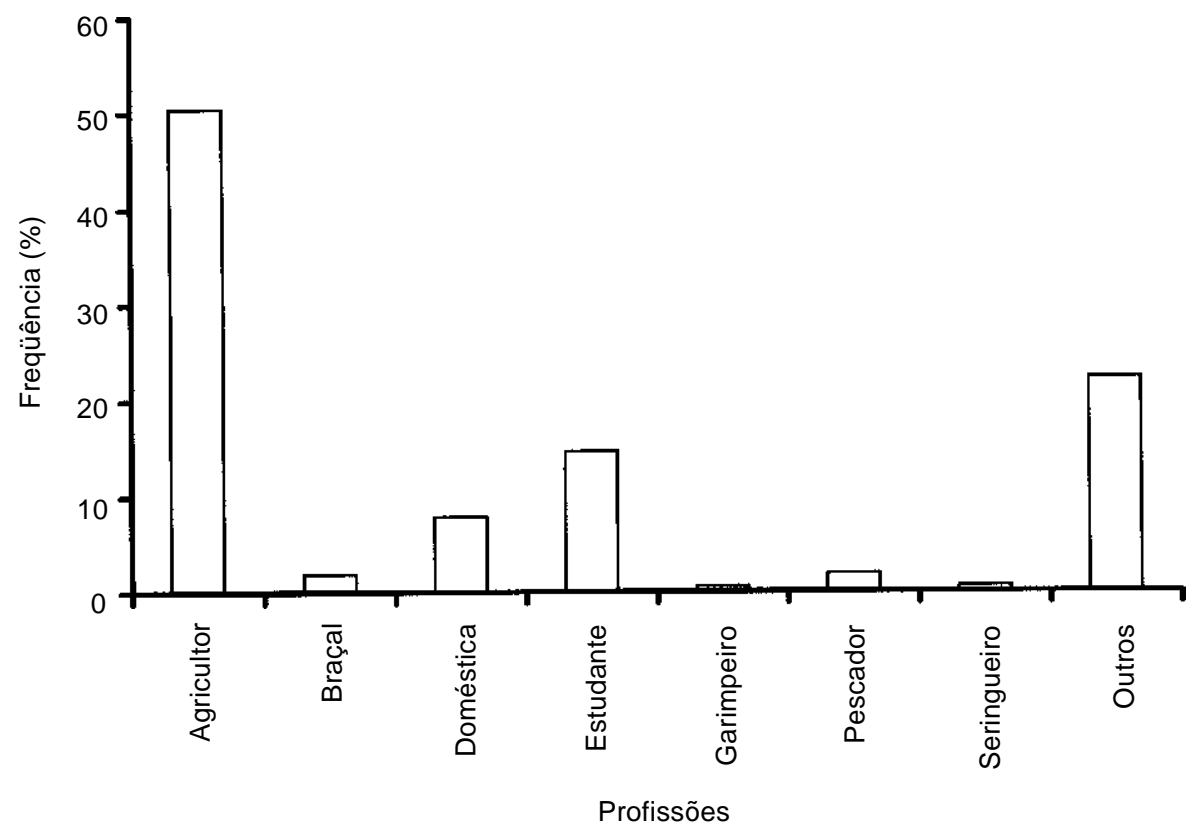

Figura 1 - Distribuicão dos pacientes acidentados por serpentes de acordo com a atividade econômica $(n=804)$ nos Municípios do Amazonas 1989 a 1996.

(72,1\%), que foi picado no membro inferior (88,5\%), por jararaca $(48,6 \%)$ ou surucucu $(46,8 \%$, Figura $2)$, durante o dia $(76,3 \%)$, na zona rural de sua cidade $(70,2 \%)$ e que só recebeu atendimento médico em tempo superior a 6 horas, após o acidente $(57,3 \%$,Figura 3$)$.

Os sintomas locais $(n=966)$, que ocorreram com maior freqüência foram edema $(76,9 \%)$ e dor $(68,7 \%)$, seguidos por eritema $(10,2 \%)$, hemorragia $(9,3 \%)$, flictena $(6,6 \%)$ e equimose $(3,1 \%)$. Dentre os sintomas sistêmicos $(n=966)$, a hemorragia (gengivorragia, epistaxe, hematêmese e hematúria) foi descrita em $18,8 \%$ dos pacientes. As freqüências para os sintomas sugeridos como diferenciais entre os acidentes laquético e botrópico ${ }^{1} 1214$ foram: vômito $(6,2 \%)$, tontura $(4,6 \%)$, náusea $(4,1 \%)$, dores abdominais $(2,4 \%)$, alterações visuais (1,9\%) e desmaio (0,9\%).

Dentre os sintomas menos freqüentes apresentados pelos pacientes, destacam-se dor abdominal $(2,4 \%)$, cianose $(2,2 \%)$ e mialgia $(1,7 \%)$. 


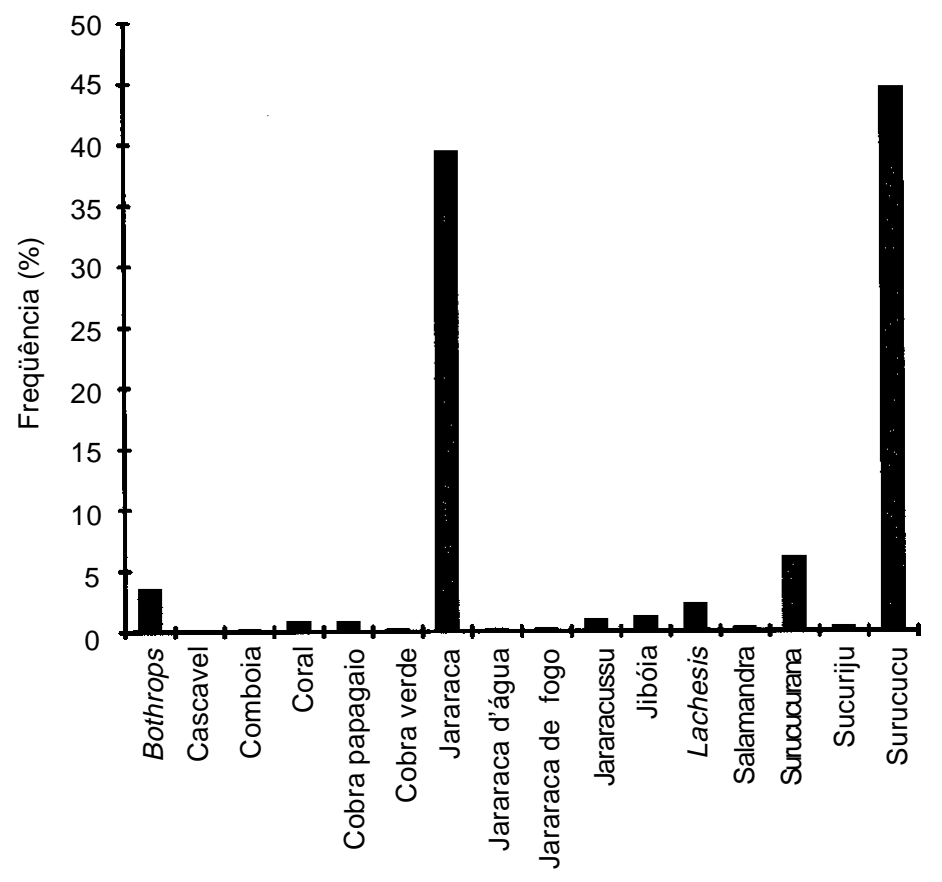

Serpentes causadoras de acidentes

Figura 2 - Serpentes causadoras dos acidentes, segundo informações de pessoas não especializadas $(n=806)$.

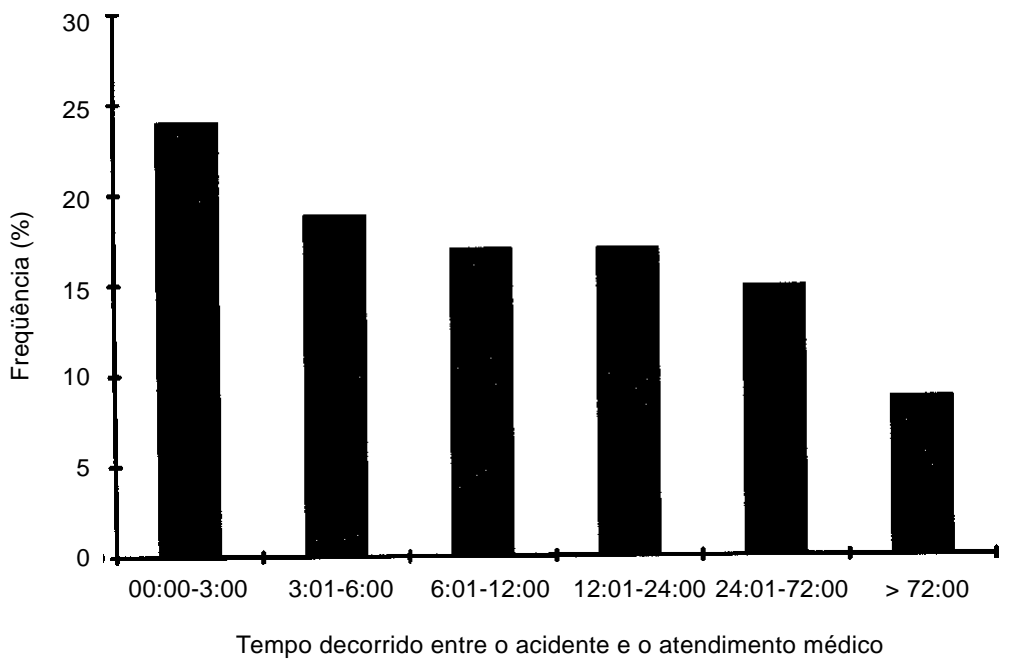

Apesar dos poucos registros da mensuração da pressão arterial $(n=288)$, a hipotensão foi aferida em $27,4 \%$ dos acidentados e a hipertensão em 5,2\%. A hipotensão foi observada, com maior freqüência, em pacientes atendidos nas
Unidades de Saúde dos municípios de Parintins, Coari e Eirunepé; e a hipertensão, nos pacientes atendidos em Parintins e Itacoatiara. Dos registros de temperatura corporal, a hipertermia (temperatura $\geq a 7^{\circ} \mathrm{C}$ ) ocorreu em $56,6 \%$ dos casos. 
O exame laboratorial para avaliar o tempo de coagulação sangüínea (TC) só foi realizado em $14 \%(n=221)$ dos pacientes. Destes pacientes, $53,8 \%$ apresentaram tempo de coagulação normal (< 10 minutos), 23,7\% alterado (entre 10 e 30 minutos) e $22,5 \%$ incoagulável (> 30 minutos).

Soroterapia e tratamento de apoio. A soroterapia foi administrada em apenas $65,9 \%$ dos pacientes $(n=1359)$. O tratamento soroterápico foi realizado com maior freqüência nos pacientes atendidos nas Unidades de Saúde dos municípios de Tabatinga (94,4\%) e São Gabriel da Cachoeira (92,3\%); e as menores freqüências foram registradas para as Unidades de Saúde de Novo Airão (26,3\%), Urucará $(47,8 \%)$ e Parintins $(49,3 \%)$.

As vias para a administração do antiveneno foram a endovenosa $(52,3 \%)$, intramuscular $(26,3 \%)$, subcutânea $(8,3 \%)$ e associações dessas vias $(13,1 \%)$.

O teste de sensibilidade ao antiveneno (soro heterólogo) foi solicitado para $16,4 \%(n=258)$ dos pacientes.

Poucos registros $(n=96)$ foram encontrados sobre a ocorrência ou não de reações alérgicas apresentadas pelos pacientes, após administração do soro heterólogo. Destes registros, $15,6 \%$ dos pacientes apresentaram hipersensibilidade aos anticorpos eqüíneos.

$\mathrm{O}$ antiveneno mais administrado foi o antibotrópico $(66,7 \%)$.

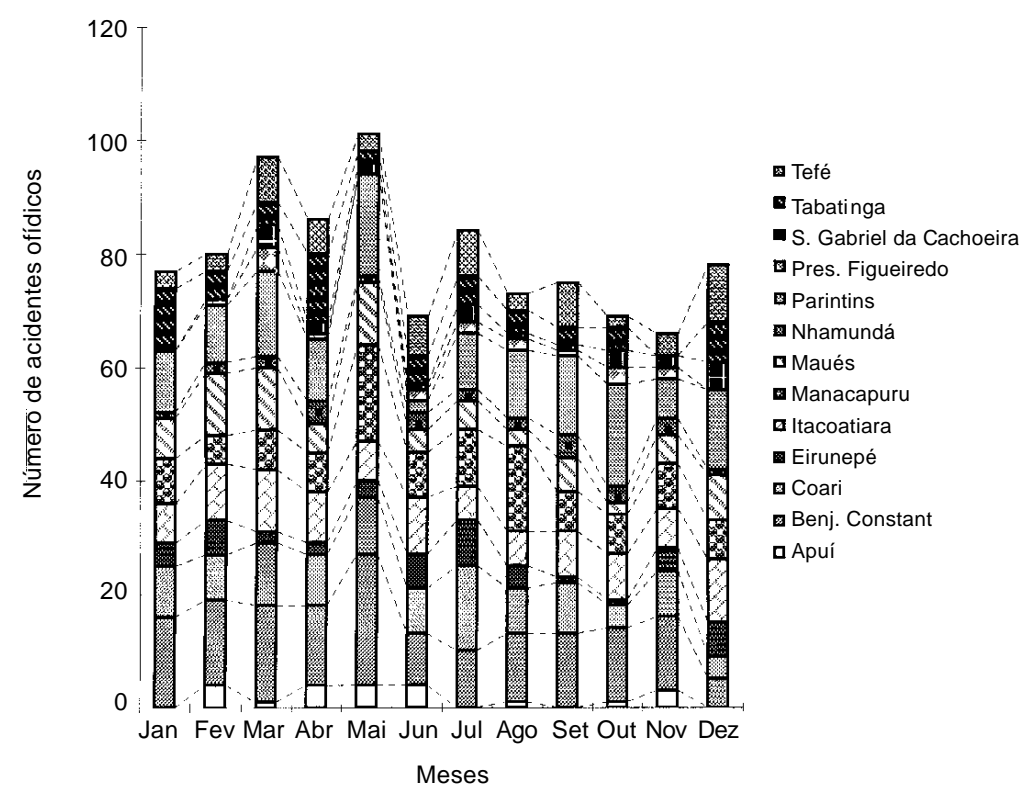

Figura 4 - Sazonalidade do acidente ofídico em treze municípios do Estado do Amazonas.
Na terapia de apoio, os antibióticos foram administrados em $73,6 \%$ dos pacientes; os analgésicos, em $61,5 \%$; os anti-histamínicos, em 40,6\%; os corticóides, em 27,3\%; as vitaminas, em 27,6\%; os anti-inflamatórios, em $26,5 \%$; o metronidazol, em 16,4\%; o toxóide tetânico, em $16,9 \%$ e, o Específico P. Pessoa, em 0,9\%.

Tempo de Internação e Alta Hospitalar. O período de internação dos pacientes variou de um até 45 dias. Porém, a maioria dos pacientes $(67,2 \%)$ ficou internada de um a cinco dias.

Poucas informações sobre as condições ou tipo de alta dos pacientes estavam disponíveis $(n=536)$. Destas, $54,5 \%$ dos pacientes tiveram alta melhorados; $32,2 \%$, curados; $6 \%$, transferidos; $5,2 \%$, a pedido; $1,1 \%$, sem autorização; e 1,0\%, óbito.

Complicações e Condutas Médico-cirúrgicas. Foram poucas as informações sobre a ocorrência de complicações $(n=204)$ e as respectivas condutas médico-cirúrgicas $(n=160)$. Dos casos analisados ocorreu abcesso local em 13,7\%, necrose em $12,3 \%$, infecção secundária em 8,3\%, insuficiência renal em $2,5 \%$, gangrena em $2,5 \%$, doença do soro em $0,5 \%$, elefantíase em $0,5 \%$ e em $59,7 \%$ não ocorreram complicações. As condutas médico-cirúrgicas utilizadas, para as complicações, foram: drenagem $(52,6 \%)$, debridamento $(28,9 \%)$, amputação (10,5\%), limpeza cirúrgica (5,3\%), diálise peritoneal $(2,6 \%)$ e fasciotomia $(2,6 \%)$.

Sazonalidade do acidente ofídico. A influência da sazonalidade (Figura 4) foi observada em 
treze municípios. Apuí apresentou maior ocorrência de acidentes em fevereiro, abril, maio e junho; Maués, em fevereiro, março e maio; Nhamundá, em abril e setembro; Parintins, em maio e outubro; Presidente Figueiredo, em março; Itacoatiara, em março e dezembro;
Tabatinga, em abril; São Gabriel da Cachoeira e Tefé em dezembro; Coari e Eirunepé, em julho; Manacapuru e Benjamim Constant em maio. A soma dos casos apresentados mostra que no mês de maio ocorreu o maior número de acidentes, seguido pelos meses de março, julho e abril.

\section{DISCUSSÃO}

Os dados referentes a sexo, idade, profissão do paciente, horário do acidente, procedência e segmento corporal picado, foram semelhantes aos encontrados em outros trabalhos realizados nas Regiões Amazônica9121925 e Sudeste ${ }^{623}$.

Considerando-se as descrições realizadas pelos médicos, pacientes ou acompanhantes, para o reconhecimento da serpente causadora dos acidentes, o somatório dos registros dos acidentes atribuídos a jararaca, ou a surucucurana e ou Bothrops correspondeu a 48,6 (Figura 2). Enquanto, o somatório dos registros para a surucucu (nome popular dado a Lachesis muta muta) ou Lachesis, resultou em 46,8\%. Em Belém (PA, Brasil) Pardal e colaboradores ${ }^{20}$, utilizando-se também das informações de pacientes e acompanhantes encontraram os seguintes resultados: a surucucu e a jararaca foram as principais serpentes causadoras de acidentes, correspondendo a $36,7 \%$ e $18,9 \%$, respectivamente. Estes dados, contrariam os resultados obtidos, no Instituto de Medicina Tropical do Amazonas (Manaus, AM, Brasil) ${ }^{25}$, pelos registros dos pacientes que trouxeram o animal causador, demonstrando ser a Bothrops atrox a principal serpente causadora de acidentes, em $76 \%$ dos casos e a Lachesis muta muta, em apenas $10 \%$.

Importante lembrar que no Amazonas existem, além da Bothrops atrox para qual são atribuídos a maioria dos acidentes na região Amazônica, mais quatro espécies de serpentes, popularmente, chamadas de jararacas (Bothrops brazilii, Bothriopsis taeniata, B. bilineata e Porthidium hyoprora). Os trabalhos realizados no Amazonas sobre acidente ofídico foram realizados, no Instituto de Medicina Tropical do Amazonas (Manaus, AM, Brasil) onde são atendidos os pacientes procedentes, principalmente, dos municípios circunvizinhos e da periferia da cidade de Manaus.

OAmazonas é o maior Estado da Federação, apresenta diversidade de ecossistemas como, matas inundadas, várzeas, igapó, terra-firme e por isso, deve ser pouco provável que apenas a Bothrops atrox seja a serpente causadora de acidentes. Estes dados, descritos a seguir dão suporte a esta hipótese, por exemplo: os acidentes causados por cobra-papagaio (nome popular dado para as serpentes Bothriopsis bilineata e Corallus caninus) foram registrados em Benjamim Constant (três acidentes), Coari (um acidente) e Manacapuru (dois acidentes), municípios localizados às margens do Rio Solimões; e os pacientes que apresentaram com maior freqüência os sintomas descritos como diferenciais para Lachesis muta muta foram os atendidos nas Unidades de Saúde de Manacapuru, Coari, Tefé (municípios localizados na margem do Rio Solimões), Itacoatiara, Parintins (municípios localizados na margem do Rio Amazonas), Maués e Urucará.

Além da diferenciação dos ecossistemas a distribuição geográfica das serpentes na Amazônia brasileira, principalmente, nos Estados do Amazonas e Acre é praticamente desconhecida, baseando-se apenas em coletas esparsas. Dessa forma, estudos sobre a freqüência de espécies e sobre estrutura das comunidades de serpentes devem ser realizados para que possamos conhecer melhor as espécies responsáveis pelos acidentes ofídicos e com isso, exigir que sejam produzidos antivenenos mais específicos para as serpentes dessa região.

Na Região Amazônica são predominantes os acidentes por serpentes dos gêneros Bothrops e Lachesis, que provocam sintomatologias semelhantes, pois sintomas vagomiméticos, descritos como diferenciais, foram presentes em apenas $14,3 \%$ dos pacientes acidentados por Lachesis muta muta ${ }^{26}$. Por este motivo, o antiveneno botrópico-laquético deveria ser administrado em pacientes que não levam a serpente causadora do acidente ao serviço médico, pois o soro antibotrópico não neutraliza, eficazmente, a atividade coagulante do veneno laquético ${ }^{2}$. No entanto, apenas $5,3 \%$ dos pacientes receberam o soro bivalente, o antibotrópicolaquético.

O número de ampolas utilizadas nos tratamentos variou de zero a quinze, sendo a 
moda o uso de cinco ampolas por acidente $(35,1 \%)$. Anão realização da soroterapia $(34,1 \%)$ e da administração de subdoses (20\%) estão relacionadas, principalmente, com a falta de antivenenos nas Unidades de Saúde. A Unidade de Saúde de Tabatinga, para minimizar esse problema, compra os antivenenos produzidos em Bogotá (Colômbia).

Outro fato importante observado foi a administração dos antiveneno pelas vias intramuscular $(26,3 \%)$, subcutânea $(8,3 \%)$ e associação de vias $(13,1 \%)$, não mais recomendadas pelo Ministério da Saúde ${ }^{1116}$ por serem menos eficazes, na neutralização dos venenos $^{22}$, que a via endovenosa (utilizada em $52,3 \%)$.

Nos casos de acidentes ofídicos, a análise do tempo de coagulação sangüínea (TC) é muito importante tanto para o diagnóstico do acidente como, também, para avaliar a eficácia da soroterapia ${ }^{181116}$. Neste levantamento o exame do tempo de coagulação foi solicitado, apenas, para $14 \%$ dos pacientes.

Os medicamentos usados no tratamento de apoio variaram entre as Unidades de Saúde do Interior do Amazonas. O maior índice de uso de antibióticos ocorreu em Parintins $(91,2 \%)$ e 0 menor, em Urucará $(23,9 \%)$.

Dos pacientes medicados com vitaminas $(\mathrm{n}=311), 26,1 \%$ receberam vitamina $\mathrm{K}$ como coadjuvante no tratamento ou na prevenção de hemorragias. Sabe-se que a vitamina K participa da síntese da protrombina e que os venenos botrópicos e laquético, na sua maioria, possuem enzimas que clivam o fibrinogênio direta e indiretamente levando ao consumo total desse fator da coagulação sangüínea ${ }^{1116}$. Portanto, o uso de vitamina $\mathrm{K}$ nesses pacientes, em nada contribui para evitar ou tratar as hemorragias causadas pelos envenenamentos ofídicos.

O metronidazol foi utilizado em $16,4 \%(n=258)$ dos acidentados e com maior freqüência, nos pacientes atendidos nas Unidades de Saúde de Urucará $(71,4 \%)$ e Autazes $(58,8 \%)$. Dos 185 pacientes que fizeram uso de metronidazol, 49 $(26,5 \%)$ receberam, também, antibiótico e não receberam soroterapia. Podendo, nestes casos, o metronidazol estar sendo utilizado, empiricamente, como substituto dos antivenenos.

O Específico P. Pessoa é um extrato vegetal usado, popularmente, como antiveneno porém, comprovadamente, sem eficácia na neutralização do veneno de Bothrops atrox ${ }^{5}$. Este extrato vegetal foi prescrito por profissionais das Unidades de Saúde dos municípios de Silves (33,3\%), Boa Vista do Ramos (20\%), Autazes $(14,7 \%)$, Maués $(2,5 \%)$ e Humaitá $(1,7 \%)$.

A percentagem de pacientes atendidos em tempo igual ou superior a 6 horas após o acidente foi de $57,3 \%$ (Figura 3), sendo semelhante à obtida em Belém (PA) que foi de $56,1 \%{ }^{20}$, mais elevada se comparada com as percentagens encontradas em Manaus (AM) ${ }^{25}$ e Letícia (Colômbia) $)^{12}$ que foram, respectivamente, 50\% e 47\%; e, consideravelmente, superior à obtida em São Paulo (SP) que foi de $12 \%{ }^{24}$. Essa alta percentagem é justificada pela dificuldade de deslocamento da zona rural, onde ocorre a maioria dos acidentes $(70,2 \%)$, para a sede dos municípios, devido a grande extensão territorial destes e a predominância do transporte fluvial no interior do Estado. Esse retardo no atendimento influencia na morbidade e letalidade dos pacientes.

$O$ quadro dos acidentados por serpentes peçonhentas, no Amazonas, é agravado, também, pela conduta assumida por parte dos profissionais da saúde, de não realizar a soroterapia em pacientes que chegam ao serviço médico em tempo igual ou superior a seis horas após a picada, isso sem qualquer fundamentação científica. Os resultados obtidos neste trabalho confirmam esta prática, pois, do cruzamento das variáveis, tempo decorrido entre o acidente e atendimento médico com soroterapia, foi observado que $76 \%$ dos pacientes que não receberam o antiveneno foram atendidos em tempo superior a seis horas, após o acidente. Esta conduta prejudica o paciente, pois o veneno mantém-se circulante por vários dias após a picada $^{3}$. O resultado do cruzamento das variáveis tempo decorrido entre acidente e atendimento médico com complicações locais e sistêmicas mostra, que o aumento das complicações do Acidente Ofídico está relacionado com a demora da chegada do paciente ao serviço médico. Dos pacientes que foram a óbito, com informação sobre o tempo decorrido entre o acidente e o atendimento, $90 \%$ chegaram em tempo superior a seis horas do acidente. Resultados semelhantes foram observados por Ribeiro e colaboradores ${ }^{23}$ onde mostraram haver uma associação entre a letalidade com o tempo decorrido entre a picada e o atendimento médico, em acidentados no Estado de São Paulo.

Do cruzamento das variáveis soroterapia com letalidade, observamos que em $33,3 \%$ dos casos de óbito os pacientes não receberam soroterapia. 
Dos que foram tratados com o antiveneno $20 \%$ receberam subdoses (1/2 a 3 ampolas). Essa situação de retardo ou de ausência da soroterapia $(34,1 \%)$, favorece a ocorrência de complicações locais e sistêmicas, com sensíveis reflexos na taxa de letalidade do acidente ofídico no Estado do Amazonas (1\%), que foi alta, considerando a predominância de acidentes botrópicos e laquéticos. A percentagem total de letalidade registrada para o Estado de São Paulo foi de $0,4 \% \%^{24}$. Ressalta-se ainda que, no Estado de São Paulo, ocorrem acidentes com cascavéis (Crotalus durissus terrificus), o gênero responsável pelo maior coeficiente de óbito no Brasil $(2,52 \%)^{15}$.
Existem trabalhos ${ }^{19}{ }^{25}$ que atribuem às cheias dos rios a variação sazonal do acidente ofídico no Estado do Amazonas. Como podemos notar (Figura 4), não há uma uniformidade entre os municípios, quanto aos meses de maior ocorrência dos acidentes ofídicos. Os dados mostram que além de uma possível influência das cheias dos rios na elevação do número de casos, deve-se considerar também a influência das atividades sócio-econômicas de cada município ou região, em especial, as agrícolas e extrativistas.

\section{AGRADECIMENTOS}

Agradecemos em especial à Força Aérea Brasileira pelo apoio de transporte e pelo auxílio nos levantamentos dos dados. Os autores agradecem ainda os integrantes do Grupo de Assistência ao Acidente Ofídico (GAAO) pelo levantamento dos dados, nas Unidades de Saúde dos Municípios.

\section{REFERÊNCIAS BIBLIOGRÁFICAS}

1. Amaral CFS, Dourado HV, Kouyoundjian JA, Cardoso JLC, Campos JA, Azevedo-Marques MM, Lopes PFA. Manual de diagnóstico e tratamento de acidentes ofídicos. Brasília: Ministério da Saúde do Brasil, 1991.

2. Bard R, Lima JCR, Sá-Neto RP, Oliveira SG, Dos-Santos MC. Ineficácia do antiveneno botrópico na neutralização da atividade coagulante do veneno de Lachesis muta muta. Relato de caso e comprovação experimental. Revista do Instituto de Medicina tropical de São Paulo 36:77-81,1994.

3. Barral-Netto M, Schriefer A, Barral A, Almeida AR, Mangabeira A. Serum levels of bothropic venom in patients without antivenom intervention. American Journal Tropical Medicine and Hygiene 45:751-754, 1991.

4. Boechat AL, Paiva CS, Dos-Santos MC Evaluation of heparin in the neutralization of main biological activities of Bothrops atrox venom (coagulant, defibrinating, edematogenic, hemorrhagic and lethal). Toxicon 36:14, 1996.

5. Borges CC, Cavalcanti-Neto AJ, Boechat AL, Franciscon $\mathrm{CH}$, Arruda LFMR, Dos-Santos MC. Eficácia da espécie vegetal Peltodon radicans (paracari) na neutralização da atividade edematogênica e a ineficácia do extrato vegetal Específico Pessoa na neutralização das principais atividades do veneno de Bothrops atrox. Revista da Universidade do Amazonas. Série: Ciências Biológicas 1:97-113, 1996.

6. Caiaffa WT, Antunes CMF, Oliveira HR, Diniz CR. Epidemiological and clinical aspects of snakebite in Belo Horizonte, Southeast Brazil. Revista do Instituto de Medicina tropical de São Paulo 39:113-118, 1997.

7. Campbell J A, Lamar WW. The Venomous Reptiles of Latin America. Ithaca: Cornell University Press, 1989.
8. Cardoso JLC, Bucaretchi F, França FOS, Puorto G, Ribeiro LA, Azevedo-Marques M M, Jorge M T, Cupo P, Moraes RHP, Hering SE, Lucas SM, Gualtieri VBF. Acidentes por Animais Peçonhentos: Identificação, Diagnóstico e Tratamento. Manual de Vigilância Epidemiológica. São Paulo: Secretaria de Estado da Saúde, 1993.

9. Castro SS, Dos-Santos MC, Arakian KL, Muniz EG, Bührnheim PF. Estudo epidemiológico dos acidentes ofídicos atendidos no Instituto de Medicina Tropical de Manaus - Amazonas, de 1986 à 1991. In: Anais do I Simpósio de Medicina Tropical do Amazonas, 1, Manaus. p. 37, 1992.

10. Cavalcanti-Neto AJ, Borges CC, Dos-Santos MC. Ineficácia do Específico Pessoa na neutralização das prinipais atividades biológicas do veneno de Bothrops atrox. Revista da Sociedade Brasileira de Medicina Tropical 28 (supl I): 308, 1995.

11. Dos-Santos MC, Martins M, Boechat AL, Sá-Neto RP, Oliveira ME. Serpentes de interesse médico da Amazônia, UA/SESU, 1995.

12. Haad JS. Accidentes Humanos por las Serpientes de los Gêneros Bothrops y Lachesis. Memórias do Instituto Butantan 44/45:403-423, 1980/81.

13. Heneine LGD, Catty D. Species-specific detection of venom antigens from snakes of the Bothrops and Lachesis genera. Toxicon 31:591-603, 1993.

14. Jorge MT, Sano-Martins IS, Tomy SC, Castro SCB, Ferrari RA, Ribeiro LA, Warrell DA. Snakebite by the bushmaster (Lachesis muta) in Brazil: case report and review of the literature. Toxicon 35:545-554, 1997.

15. Ministério da Saúde do Brasil. Ofidismo, análise epidemiológica. Brasília, 1994. 
16. Ministério da Saúde do Brasil e Fundação Nacional da Saúde. Manual de Diagnóstico e Tratamento de Acidentes por Animais Peçonhentos. Brasília, 1998.

17. Nahas L, KamigutiAS, Rzeppa HW, Sano IS, Matsunaga S. Effect of Heparin on The Coagulan Action of Snake Venomous. Toxicon 13: 457-463, 1975.

18. Olortegui CC, Lopes CS, Cordeiro FD, Granier C, Diniz CR. An enzyme linked immunosorbent assay (ELISA) that discriminates between Bothrops atrox and Lachesis muta muta venoms. Toxicon 31:417-425, 1993.

19. Pardal PPO, Monteiro MRCC, Arnaund RN, Lopes FOB, Asano ME. Aspectos epidemiológicos de 465 acidentes ofídicos atendidos no HUJBB - Belém - Pará no período de 1993 a 1994. Revista da Sociedade Brasileira de Medicina Tropical 28 (supl I):170, 1995.

20. Pardal PPO, Asano ME, Arnaund RM, Lopes FOB, Monteiro MRCC. Aspectos Clínicos e complicações de 465 acidentes ofídicos atendidos no HUJBB-Belém-Pará no período de 1993-1994. Revista da Sociedade Brasileira de Medicina Tropical 28 (supl 1):172, 1995.

21. Praxedes LA, Gonçalves LRC, Souza e Silva MCC, Cury Y, Kelen E. Metronidazole and anti-Bothrops (ABS) association: effect on Bothrops jararaca $(\mathrm{Bj})$ venominduced edema and local hemorrhage. Toxicon 36:29, 1996.
22. Ribeiro LA, Agostini-Utescher CL, Vieira SLP, Fensterseifer S, Mukuno H, Jorge MT. Avaliação em camundongos da eficácia do antiveneno administrado no local da inoculação intramuscular do veneno de Crotalus durissus terrificus. Revista do Instituto de Medicina tropical de São Paulo 35:23-27, 1993.

23. Ribeiro LA, Albuquerque MJ, Campos VAFP, Katz G, Takaoka NY, Lebrão ML, Jorge MT. Óbitos por serpentes peçonhentas no Estado de São Paulo: avaliação de 43 casos, 1988/93. Revista da Associação Médica Brasileira 44:312-318, 1998.

24. Ribeiro LA, Campo VAFP, Albuquerque MJ, Takaoka NY. Acidente Ofídico no Estado de São Paulo. Revista da Associação Médica Brasileira 39:4-7, 1993.

25. Sá-Neto R P, Dos-Santos MC. Aspectos epidemiológicos dos acidentes ofídicos atendidos no Instituto de Medicina Tropical de Manaus (IMTM), 1986-92: estudo retrospectivo. Revista da Sociedade Brasileira de Medicina Tropical 28 (supl I):171,1995.

26. Sá-Neto RP, Dos-Santos MC. Aspectos clínicos comparativos do acidente botrópico e laquético. Revista Sociedade Brasileira de Medicina Tropical 28 (supl I):173, 1995.

27. Tinoco RC. Picada de Cobra (Uso de Heparina como Substituto de Soro Antiofídico). Jornal Brasileiro Medicina 23:109-114, 1972. 